\title{
Simulated Trading Mechanismen für Speditionsübergreifende Transportplanung
}

\author{
Hans-Jürgen Bürckert, Gero Vierke
}

December 1998

\section{Deutsches Forschungszentrum für Künstliche Intelligenz $\mathrm{GmbH}$}

Postfach 2080

67608 Kaiserslautern, FRG

Tel.: + 49 (631) 205-3211

Fax: + 49 (631) 205-3210

E-Mail: info@dfki.uni-kl.de
Stuhlsatzenhausweg 3

66123 Saarbrücken, FRG

Tel.: + 49 (681) 302-5252

Fax: + 49 (681) 302-5341

E-Mail: info@dfki.de

WWW: http://www.dfki.de 


\section{Deutsches Forschungszentrum für Künstliche Intelligenz DFKI GmbH German Research Center for Artificial Intelligence}

Founded in 1988, DFKI today is one of the largest nonprofit contract research institutes in the field of innovative software technology based on Artificial Intelligence (Al) methods. DFKI is focusing on the complete cycle of innovation - from world-class basic research and technology development through leading-edge demonstrators and prototypes to product functions and commercialization.

Based in Kaiserslautern and Saarbrücken, the German Research Center for Artificial Intelligence ranks among the important "Centers of Excellence" worldwide.

An important element of DFKl's mission is to move innovations as quickly as possible from the lab into the marketplace. Only by maintaining research projects at the forefront of science can DFKI have the strength to meet its technology transfer goals.

DFKI has about 115 full-time employees, including 95 research scientists with advanced degrees. There are also around 120 part-time research assistants.

Revenues for DFKI were about 24 million DM in 1997, half from government contract work and half from commercial clients. The annual increase in contracts from commercial clients was greater than $37 \%$ during the last three years.

At DFKI, all work is organized in the form of clearly focused research or development projects with planned deliverables, various milestones, and a duration from several months up to three years.

DFKI benefits from interaction with the faculty of the Universities of Saarbrücken and Kaiserslautern and in turn provides opportunities for research and Ph.D. thesis supervision to students from these universities, which have an outstanding reputation in Computer Science.

The key directors of DFKI are Prof. Wolfgang Wahlster (CEO) and Dr. Walter Olthoff (CFO).

DFKI's six research departments are directed by internationally recognized research scientists:

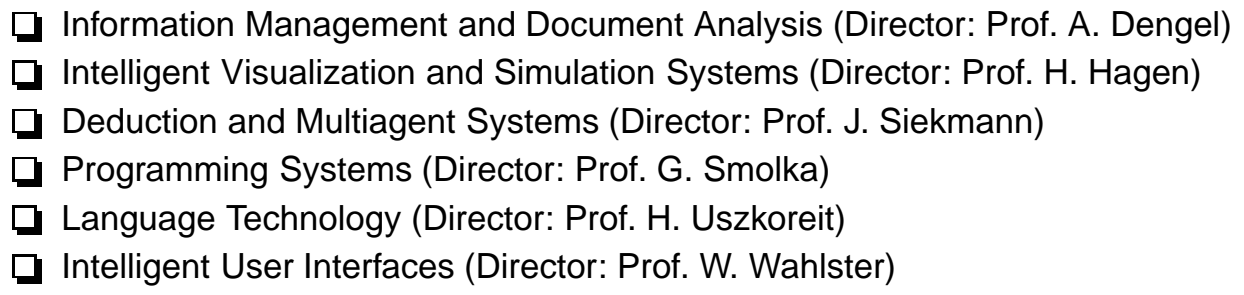

In this series, DFKI publishes research reports, technical memos, documents (eg. workshop proceedings), and final project reports. The aim is to make new results, ideas, and software available as quickly as possible.

Prof. Wolfgang Wahlster

Director 
Simulated Trading Mechanismen für Speditionsübergreifende Transportplanung

Hans-Jürgen Bürckert, Gero Vierke

DFKI-TM-98-04 


\section{Diese Arbeit wurde durch die Regierung des Saarlandes gef̈rdert.}

(C) Deutsches Forschungszentrum für Künstliche Intelligenz 1998

This work may not be copied or reproduced in whole or part for any commercial purpose. Permission to copy in whole or part without payment of fee is granted for nonprofit educational and research purposes provided that all such whole or partial copies include the following: a notice that such copying is by permission of the Deutsche Forschungszentrum für Künstliche Intelligenz, Kaiserslautern, Federal Republic of Germany; an acknowledgement of the authors and individual contributors to the work; all applicable portions of this copyright notice. Copying, reproducing, or republishing for any other purpose shall require a licence with payment of fee to Deutsches Forschungszentrum für Künstliche Intelligenz. 


\title{
Simulated Trading Mechanismen für Speditionsübergreifende Transportplanung
}

\author{
Hans-Jürgen Bürckert, Gero Vierke \\ Deutsches Forschungszentrum für Künstliche Intelligenz (DFKI GmbH) \\ Stuhlsatzenhausweg 3, D-66123 Saarbrücken \\ $\{$ hjb,vierke\}@dfki.de \\ Dezember 1998
}

\section{Zusammenfassung}

In den letzten Jahren hat sich der Konkurrenzdruck, unter dem die kleinen und mittleren Unternehmen der deutschen Speditionsbranche stehen, maßgeblich verschärft. Eine Möglichkeit, diesem Druck zu begegnen, ist die Bildung von Speditionsverbünden, sozusagen virtuellen Transportunternehmen, die in der Lage sind, durch kooperative Disposition, ihre Wettbewerbsfähigkeit zu erhöhen.

In diesem Papier stellen wir einen Agentenbasierten Ansatz zur IT Unterstützung der Unternehmensabläufe, insbesondere der Transportoptimierung, in einem solchen virtuellen Transportunternehmen vor. Der verteilte, marktbasierte Simulated Trading Optimierungsmechanismus liefert gute Ergebnisse in kooperativen Multiagenten-Planungsszenarien. Wir haben den Mechanismus um die Möglichkeit des expliziten, monetären Nutzentransfers zwischen den Beteiligten Agenten ergänzt. Somit können wir auch in kompetitiven Domänen effiziente Transportoptimierung vornehmen.

\section{$1 \quad$ Einführung}

In der letzten Dekade ist das Güterverkehrsaufkommen in Europa dramatisch angewachsen [Carroué 97]: Der Warenaustausch zwischen den Mitgliedsländern der EU ist um mehr als $80 \%$ gestiegen. Hinzu kommen $50 \%$ mehr Warenströme in und aus Nicht-EU-Ländern. Allein in Deutschland hat sich seit 1960 die Mobilität im Güter- und Personenverkehr verdoppelt. Weitere Steigerungen werden prognostiziert: Bis 2010 wird sich der Transitverkehr etwa verdreifachen. Deutschland wird dabei noch stärker als bisher zum Haupttransitland für Nord-Süd- und besonders für Ost-West-Transporte werden.

Eine weitere Ursache für diese Entwicklung sind Veränderungen in den Produktionsabläufen. Modernes Supply Chain Management erfordert hochflexible und zeitgenaue Zulieferung - in der Regel durch den LKW, weil der Schienengüterverkehr bisher weder preislich noch zeitlich konkurrenzfähig ist. Dadurch wurde jedoch eine Verkehrsdichte-Fahrtzeit-Spirale in Gang gesetzt, die bereits heute zu 
solch paradoxen Situationen führt, daß ein Lieferant gleich mehrere Transportfahrzeuge für ein und denselben Auftrag auf die Straße schickt, in der Hoffnung, daß wenigstens eines davon rechtzeitig ankommt. Gleichzeitig führt der harte Wettbewerb zwischen den vielen kleinen und kleinsten Fuhrunternehmen dazu, daß gleichzeitig mehrere LKW beim Empfänger jeweils nur wenige, einzelne Ladungseinheiten anliefern - oft von nahe beieinander oder auf derselben Route liegenden Versendern.

Das Resultat sind Staus auf den Autobahnen, Stop and Go im städtischen Lieferverkehr, Warteschlangen an den Laderampen und dabei mehr als die Hälfte der Fahrstrecken ohne oder mit wenig Fracht: Die durchschnittliche Auslastung eines 40-Tonners liegt bei nur 13 Tonnen, die Leerfahrtenquote liegt bei 30 Prozent [Klempp 98]. Dies ist der Alltag im Straßergüterverkehr auch noch über das Zukunftsjahr 2000 hinaus.

Nur eine deutlich verbesserte, sich auf moderne Informations- und Kommunikationstechnologien stützende Logistik und Transportplanung, die neben der sich steigernden Komplexität auch neue Wege der kooperativen, speditionsübergreifenden Transportabwicklung unterstützen kann, eröffnet Chancen für die Beherrschung des explodierenden Transportaufkommens: Überflüssige Fahrten müssen drastisch reduziert und die Transportwege selbst rigoros verkürzt oder bei Langstrecken durch modernen kombinierten Straße-Schiene-Verkehr ersetzt werden [Funk et al. 98]. Eine Änderung der derzeitigen Auftragsabwicklung - insbesondere auch bei den im harten Konkurrenzkampf stehen kleineren und mittleren Fuhrunternehmen - birgt daher ein enormes Einsparpotential sowohl für betriebs- als auch für volkswirtschaftliches Vermögen.

Eine verbreitet verfolgte, aber immer noch unzureichend umgesetzte Möglichkeit, die Kooperation - sowohl unimodal im reinen Straßentransport als auch intermodal im kombinierten Verkehr - zwischen Unternehmen im Transportgewerbe zu verbessern, liegt in der Einrichtung sogenannter logistischer Dienstleistungsund Güterverkehrszentren (LDZ/GVZ). In ihnen wird zum einen der lokale Verkehr einer Region zentral koordiniert und abgewickelt. Zum anderen werden die überregionalen Transporte gebündelt und den Anforderungen entsprechend die geeigneten Verkehrsträger ausgewählt.

Neben der Ausstattung der in solchen Zentren zusammenarbeitenden Unternehmen, ihrer Fuhrparks und ihrer Angestellten mit moderner Informations- und Telekommunitionshardware (für Mobil- und Satellitenkommunikation, Ladungsund Fahrzeugortung und -verfolgung) ist ein softwaretechnischer Ansatz erforderlich, der die Heterogenität der beteiligten Partner und Systeme berücksichtigt.

Eine vielversprechende Technologie hierfür sind die aus der Künstlichen Intelligenz (KI) stammenden Multiagentensysteme (MAS).

Ein Intelligenter Agent wird dabei durch eine in sich abgeschlossene Einheit, ein wissensbasiertes Computer-Programm, repräsentiert, die sowohl die Möglichkeit hat, Schlußfolgerungen zu ziehen, als auch über eine explizite Repräsentation des lokalen Wissens verfügt. Ein Multiagentensystem besteht aus einer Menge solcher Agenten, die miteinander kommunizieren können und auf diese Weise in der Lage sind, ein Problem kooperativ zu lösen. Ein Agent kann stellvertretend sowohl für ein physikalisches System als auch für eine abstrakte Instanz der Anwendungsdomäne eingeführt werden. 


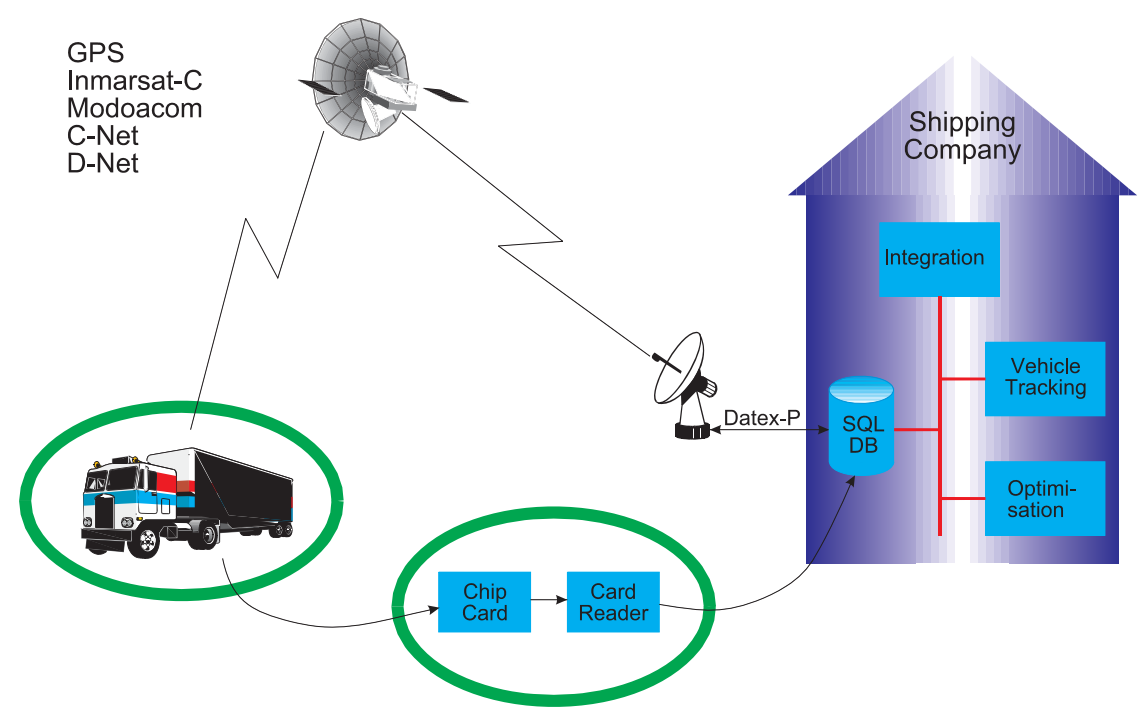

Abbildung 1: Technologien für Lösungen der Verkehrstelematik

In der Transportdomäne lassen sich z.B. für die Speditionen und für die LKW eigene Agenten definieren. Die LKW-Agenten planen selbständig ihre Route für die übernommenen Aufträge, sind verantwortlich für deren reibungslose Abwicklung und unterstützen so den Disponenten bei seiner Planungsaufgabe. Die Aufträge an die Speditionen kommen entweder von den Kunden, von fremden Speditionen, von den Partnerspeditionen oder einer Frachtbörse. Die Speditionsagenten übernehmen dabei u.a. die Koordination der Auftragszuweisung und die Datenübermittlung von Auftragseingängen an die LKW-Agenten. Sie koordinieren ferner die speditionsweite Optimierung der lokalen Planung und kooperieren mit anderen Speditionen für eine Speditionsübergreifende Planung. Ein weiterer, sehr wesentlicher Gesichtspunkt bei diesem Ansatz ist, daß die Autonomie der einzelnen Agenten gewahrt bleibt. Im Gegensatz zu einer zentralen Steuerung der Planung, obliegt bei dieser Modellierung die Entscheidung über die Annahme oder Abgabe eines Kooperationsangebots ganz allein dem einzelnen Agenten. Im folgenden wird zunächst kurz ein Dispositionsunterstützungssystem TELETruck, dessen Prototyp am DFKI für den Einsatz bei der dynamischen Transportplanung und Transportüberwachung in der Einzelspedition entwickelt wurde, vorgestellt (Abschnitt 2). Danach werden Einsatzmöglichkeiten und die dafür notwendigen Anforderungen für den speditionsübergreifenden Einsatz zunächst konzeptionell skizziert (Abschnitt 3). In den Folgeabschnitten werden dann das zentrale Optimierungsverfahren des TELETRUCK-Systems beschrieben (Abschnitt 4) und die Erweiterung auf ein interbetriebliches Optimierungverfahren vorgestellt (Abschnitt 5). Eine Diskussion dieser Methode und ein Ausblick auf die prototypische Realisierung durch verteilt eingesetzte, vernetzte Kopien des geeignet erweiterten TeleTRUCK-Systems beschließen den Beitrag. 


\section{Das TeleTruck-System}

Am DFKI wird ein multiagenten-basiertes Dispositionsunterstützungssystem, der TeleTruck-Prototyp [Bürckert et al. 98, Fischer 98] entwickelt. Es ist als interaktives System zur dynamischen Transportplanung und Überwachung der Auftragsabwicklung zunächst noch für die Einzelspedition konzipiert. Das System modelliert die Spedition selbst, ihre Fahrzeuge und deren Komponenten sowie Fahrer durch ein holonisches Multiagentensystem [Gerber et al. 99]. Das heißt, die obigen Einheiten werden im System durch intelligente Agenten repräsentiert, die über Planungs- und Interaktionsfähigkeiten für Kommunikation und Verhandlung verfügen. Die Agenten, die ganze Fahrzeuge, aber auch deren Komponenten (wie Laderaum und Hänger) und ihre Fahrer repräsentieren, sind darüber hinaus in der Lage, sich durch Selbstorganisation zu komplexeren Transport-Einheiten, holonischen Transport-Agenten ${ }^{1}$, zusammenzufinden, die dann komplette Transporte planen und deren Ausführung durch ihre physikalischen Gegenparts über entsprechende telematische Dienste (Telekommunikation und GPS, s. Abbildung 1) — überwachen können. Durch solche Dienste wird auch eine dynamische Reaktion auf Veränderungen in der Planausführung — sei es aufgrund von Verzögerungen (Staus, Wartezeiten) oder durch Änderungen von Auftragsdetails (Ladungsumfang, Lieferzeiten) oder durch den kurzfristigen Eingang weiterer Aufträge - möglich.

Eingehende Transportaufträge werden in einer zentralen SQL-Datenbank abgelegt und durch einen Speditionsagenten auf die Fahrzeuge verteilt. Dies geschieht durch Ausschreibung der Aufträge an die Transportagenten, die in einer simulierten Versteigerung über die Aufträge verhandeln. Grundlage der Verhandlung ist die lokale Planung der Transportagenten anhand der Entfernungs- oder ggf. Fahrzeitdaten aus elektronischem Kartenmaterial, ihrer bereits eingeplanten Transporte und Fahrstrecken sowie der noch verfügbaren Resourcen (Fahrerzeiten und Laderaum) und den Entfernungsdaten aus elektronischem Kartenmaterial. Letztere können - als Sub-Agenten des Transport-Holons — während der Planung ausgetauscht oder auch hinzugezogen (zweiter Fahrer oder Hänger): die Transport-Holonen organiseren und re-organisieren sich in entsprechenden nachgeschalteten Verhandlungszyklen selbständig nach Bedarf. Da durch dieses Vorgehen nur suboptimale, etwa von der Ausschreibungsreihenfolge abhängige Pläne generiert werden, können diese in einem markt-ähnlichen Tauschverfahren (Simulated Trading, s. Abschnitt 4) optimiert werden. Während solcher Tauschrunden können neue Aufträge hinzukommen oder die alten Aufträge modifiziert werden. Dies kann prinzipiell auch noch während der Auftragsausführung geschehen zumindest für Auftragsteile deren Bearbeitung noch nicht begonnen hat - wodurch die erwähnte dynamische Reaktion auf Veränderungen ermöglicht wird. Alle Planungsdaten werden in der zentralen SQL-Datenbank gespeichert.

Der Speditionsagent fungiert über obige Koordinationsaufgabe hinaus als Schnitt-

\footnotetext{
${ }^{1}$ Im Rahmen dieser Selbstorganisation geben die Agenten einen Teil ihrer AgentenAutonomie zeitweise auf und verschmelzen gewissermaßen zu einem übergeordneten Holon (Zusammensetzung aus dem griechischen Begriff holos, das Ganze, und dem griechischen Suffix - on für Teil), so daß die Gruppe nach außen hin gegenüber anderen Agenten im MAS als einzelner Agent auftritt.
} 


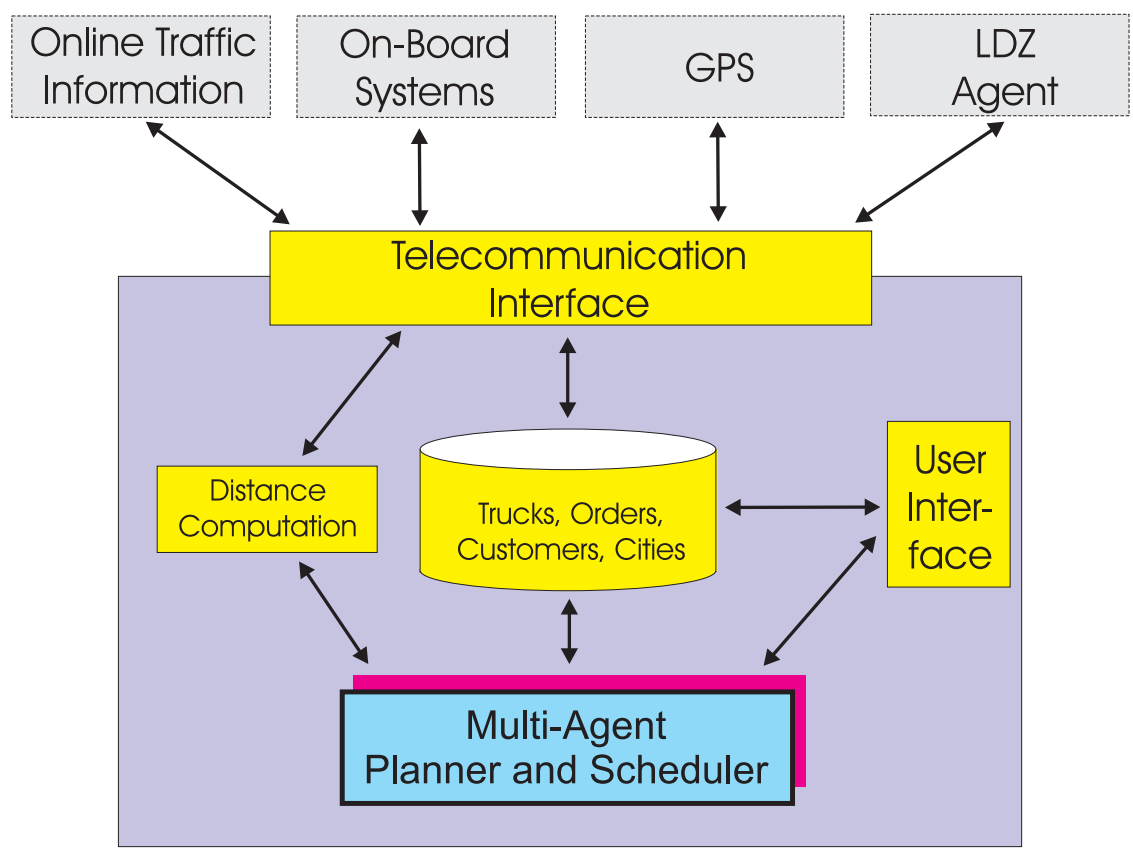

Abbildung 2: Die TeleTruck Architektur

stelle zu seinem menschlichen Gegenpart, dem für die Disposition verantwortlichen Systembenutzer ${ }^{2}$. Dieser hat so Zugriff auf die Zusammensetzung und die lokalen Pläne der Transport-Holonen und ihrer Komponenten, den Gesamtplan, die Auftragsdaten und natürlich die Stammdaten der Spedition (Kunden, Fahrzeuge etc.). In Abbildung 2 wird die Systemarchitektur mit dem zentralen holonischen MAS als Planungs- und Optimierungskomponente, den Schnittstellen zum Benutzer, den telematischen Diensten sowie zum elektronischen Kartenmaterial dargestellt.

\section{Kooperierende Speditionen eines logistischen Dienstleistungszentrums}

Am DFKI werden derzeit im Rahmen eines von der saarländischen Regierung geförderten Projektes TeleTruck-CC (für Cooperating Companies) in Zusammenarbeit mit lokalen Speditionen Kooperationsmodelle für ein logistisches Dienstleistungszentrum entwickelt, die in einem verteilten Anwendungsdemonstrator prototypisch realisiert und evaluiert werden. Die grundlegende (längerfristige) Zielsetzung ist es somit, eine Reihe eigenständiger Speditionen zu vernetzen und eine Kooperationsstruktur zu schaffen, die es ermöglicht, die Geschäftsabläufe innerhalb der Speditionen und innerhalb des Verbundes dahingehend zu optimieren, daß nicht nur der globale Gewinn des Verbundes gesteigert werden kann, sondern daß jeder einzelne Teilnehmer einen Vorteil erzielen kann.

\footnotetext{
${ }^{2}$ Von daher wäre tatsächlich die Bezeichnung Dispositionsagent besser geeignet. Der Name Speditionsagent wurde jedoch im Hinblick auf die Erweiterungen (s.u.) gewählt.
} 


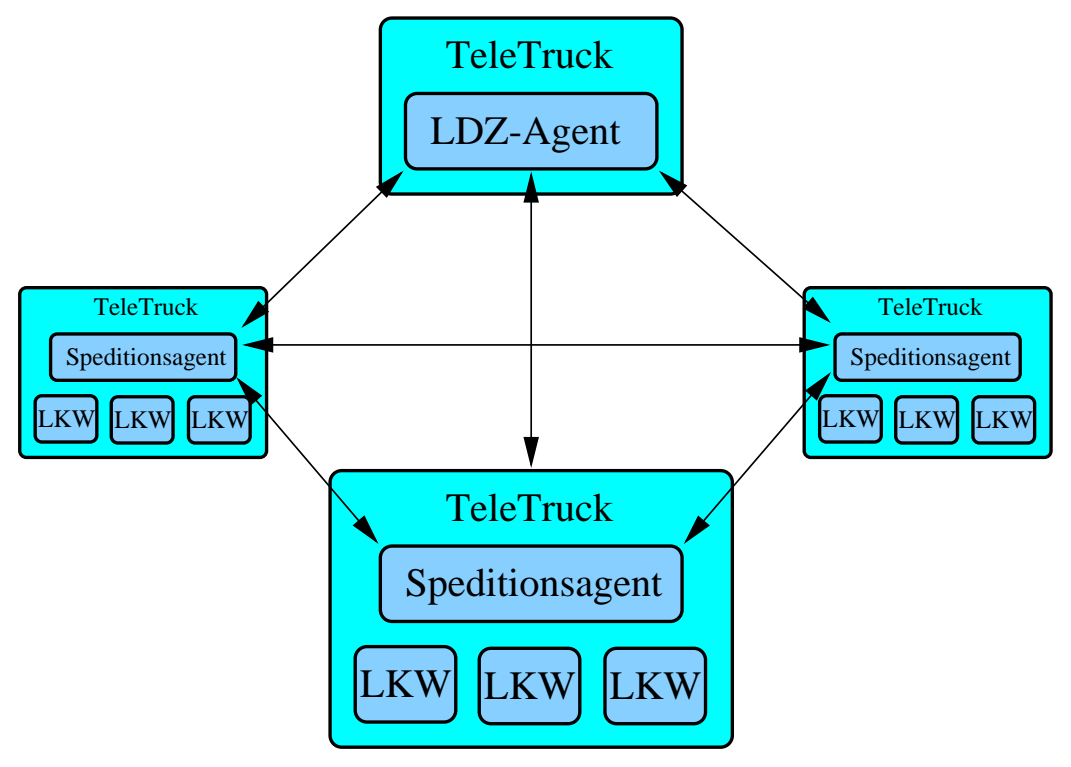

Abbildung 3: Das TeleTruCK-CC Agentensystem

Als Voraussetzung für TELETRUCK-CC sehen wir vor, die kooperierenden Speditionen mit Kopien unseres TeleTruck-Systems auszustatten, die um die zusätzliche Kooperationsfunktionalität erweitert werden. Da die TeleTruckCC-Lösung für einen Speditionsverbund entwickelt wird, der über ein LDZ koordiniert wird, ist es sinnvoll, sich nicht auf eine reine dezentrale Koordination innerhalb des Verbundes zu beschränken, sondern das Koordinationspotential, das eine zentrale Instanz ermöglichen kann, auszuschöpfen, indem die Zentrale selbst in der Agentengesellschaft modelliert wird. Abbildung 3 stellt die Struktur des Agentensystems in TeleTruck-CC dar.

Diese zentrale Instanz, der LDZ-Agent, wird im wesentlichen durch ein zusätzliches TeleTRuCK-System repräsentiert, das nicht über einen eigenen Fuhrpark verfügt, aber um die Möglichkeit, die speditionsübergreifende Optimierung zu koordinieren, erweitert worden ist. Die Modellierung der LDZ durch ein TeleTruck-System ermöglicht es auch, daß die Zentrale als gemeinsamer Ansprechpartner der verbundenen Speditionen für die Kunden auftreten kann.

Für eine speditionsübergreifende Optimierung der Disposition müssen die Speditionen dem LDZ-Agent Aufträge und ggf. Resourcen für die globale Optimierung zur Verfügung stellen. Um eine gute Gesamtlösung zu erhalten, ist es erforderlich, daß nicht nur Aufträge in die Optimierung gegeben werden, die negativen oder minimalen Gewinn einbringen, sondern auch Aufträge, die durchaus gewinnbringend bearbeitet werden könnten, die jedoch von Partner-Speditionen mit höherer Gewinnspanne durchgeführt werden können. Damit die Speditionen in so einem Fall auch solche Aufträge einbringen, muß sichergestellt werden, daß das Verkaufen eines solchen Auftrages stets profitabler ist, als ihn selbst durchzuführen. 


\section{Das Simulated Trading Verfahren}

Für die Optimierung der Disposition innerhalb einer unternehmerischen Einheit hat sich der Simulated Trading Algorithmus bewährt [Malich 94]. Hierbei wird vorausgesetzt, das bereits eine Disposition vorliegt, die optimiert werden soll. Simulated Trading modelliert einen koordinierter Markt, bei dem die LKW Aufträge austauschen (handeln) können. Die LKW-Agenten geben hierbei Kaufs- und Verkaufsgebote für Aufträge ab, die von dem koordinierenden Speditionsagenten einander so zugeordnet werden, daß die Gesamtkosten minimiert werden. Die Kosten, die ein LKW hierbei für einen Auftrag angibt, dienen lediglich als Richtlinie für den Speditionsagenten; es findet kein Geld- oder Nutzentransfer zwischen den LKW-Agenten statt, da sie Bestandteile desselben Unternehmens sind.

Durch den Verzicht auf Nutzenausgleich zwischen den LKW-Agenten wird es ermöglicht, Tauschgeschäfte abzuschließen, die für einzelne Agenten eine lokale Verschlechterung ihrer Disposition darstellen, allerdings in der Gesamtsicht eine Verbesserung bewirken. Außerdem wird es möglich, auch Tauschgeschäfte zu erlauben, die eine Verschlechterung der Gesamtlösung bewirken. Dies kann erforderlich sein, da die Menge der möglichen Lösungen für das Dispositionsproblem lokale Optima enthält: suboptimale Lösungen, die nicht durch einfache Tauschoperationen verbessert werden können, die jedoch durch mehrfache Anwendung von Tauschoperationen in günstigere Lösungen überführt werden können. Immer wenn der Speditionsagent sich entscheidet die aktuelle Lösung zu verschlechtern, wird die Lösung gesichert, für den Fall, daß im zur Verfügung stehenden Zeitrahmen keine bessere Lösung gefunden werden kann.

Die Optimierung mittels Simulated Trading findet in mehreren, aufeinanderfolgenden Runden statt. In jeder Runde werden Tauschoperationen durchgeführt, die entweder die aktuelle Lösung verbessern, oder die, wenn keine Verbesserung mehr möglich ist, kontrollierte Verschlechterungen in Kauf nehmen, um lokale Minima zu verlassen.

Eine Trading Runde besteht aus mehreren Entscheidungsebenen. Sei $A$ die Menge der an der Optimierung teilnehmenden Agenten und $O$ eine Auftragsmenge, die bereits diesen Agenten zugeordnet ist. Jeder Teilnehmer $a_{i} \in A$ gibt auf jedem Entscheidungslevel $j$ ein Gebot $b_{i j}$ ab. Die Gebote $b=((t(b), o(b), c(b))$ sind 3Tupel wobei $t(b) \in\{$ buy, sell, nop $\}$ angibt, ob es sich um ein Kauf-, ein Verkaufoder ein leeres Gebot handelt; $o(b) \in O$ bestimmt den Auftrag, auf den das Gebot sich bezieht und $c(b) \in \mathbb{R}$ sind die Kosten, die mit diesem Auftrag verbunden sind. Die Entscheidung der Agenten, welche Aufträge sie anbieten bzw. kaufen, fällt randomisiert. Die Wahrscheinlichkeit, für Gebote, die die Qualität des lokalen Plans verbessern, ist jedoch größer, als solche, die sie verschlechtern.

Ein Verkaufgebot $b_{i j}$ ist gültig, wenn dem Agenten $a_{i}$ der Auftrag $o\left(b_{i j}\right)$ zugeordnet ist, und nicht bereits auf einer früheren Entscheidungsebene angeboten wurde. Ein Kaufgebot ist gültig, wenn auf einer früheren Entscheidungsebene ein anderer Agent ein Verkaufsgebot für diesen Auftrag abgegeben hat.

Aus allen Geboten, die in einer Runde abgegeben werden, wird der Trading Graph erstellt, ein gerichteter Graph, dessen Knoten mit den Geboten beschriftet sind. Gerichtete Kanten $e\left(b_{\text {sell }}, b_{b u y}\right)$ weisen von Verkaufgeboten $b_{\text {sell }}$ zu Kaufgeboten $b_{\text {buy }}$ für denselben Auftrag $\left(o\left(b_{\text {sell }}\right)=o\left(b_{\text {buy }}\right)\right)$. Die Kanten sind mit den 


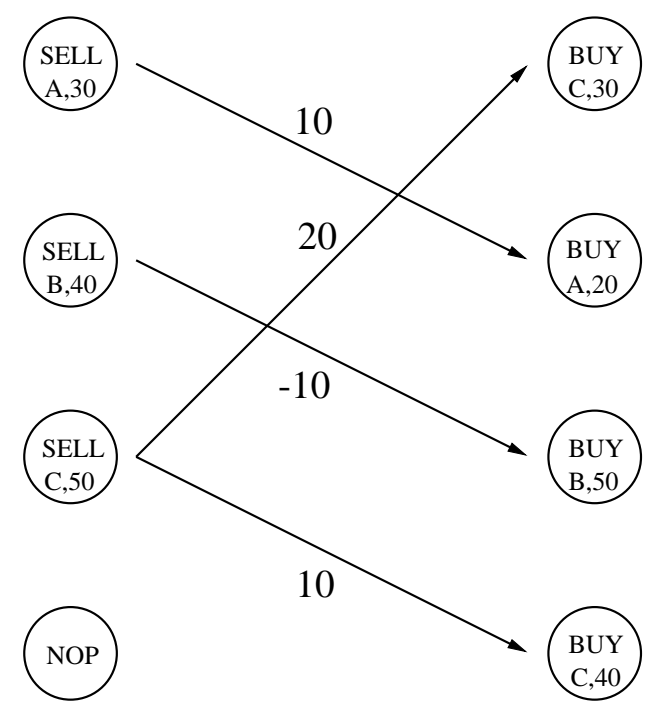

Abbildung 4: Beispiel für einen Trading Graphen

Preisdifferenzen beschriftet, die die Verbesserung (oder, falls negativ Verschlechterung) der Gesamtlösung anzeigen, falls dieser Handel zustande kommt, also $c\left(e\left(b_{\text {sell }}, b_{\text {buy }}\right)\right)=c\left(b_{\text {sell }}\right)-c\left(b_{\text {buy }}\right)$.

Die Aufgabe des Stock-Managers besteht nun darin, eine Zuordnung von Kaufund Verkaufgeboten zu finden, die die Auftragsallokation optimiert. Hierbei muß berücksichtigt werden, daß ein Verkauf maximal einem Kauf zugeordnet werden, und daß Gebote, die von Geboten auf vorherigen Entscheidungsebenen abhängen, nur akzeptiert werden können, wenn die vorherigen Gebote ebenfalls akzeptiert werden.

Es ist also eine Kantenmenge $E \mathrm{zu}$ finden, für die gilt:

- Für jedes $e\left(b_{\text {sell }}, b_{b u y}\right) \in E$ gilt; es existiert kein $b_{b u y}^{\prime} \neq b_{b u y}$, so daß $e\left(b_{\text {sell }}, b_{b u y}^{\prime}\right) \in E$.

- Für alle $e\left(b_{i j}, b_{k l}\right) \in E$ gilt: für alle $j^{\prime}<j$ ist $t\left(b_{i j^{\prime}}\right)=$ nop oder es existiert ein $b_{b u y}$ mit $e\left(b_{i j^{\prime}}, b_{b u y}\right) \in E$ bzw. ein $b_{\text {sell }}$ mit $e\left(b_{\text {sell }}, b_{i j}\right) \in E$. Analoges gilt für $b_{k l}$.

- Die Summe der Nutzen $\sum_{e \in E} c(e)$ ist maximal.

Abbildung 4 stellt einen Trading Graphen dar, der neben der trivialen Zuordnung (es finden keine Verkäufe statt) zwei Zuordnungen enthält, die die Lösung verbessern:

1. $a_{1}$ verkauft $\mathrm{A}$ an $a_{2}, a_{2}$ verkauft $\mathrm{B}$ an $a_{3}, a_{3}$ verkauft $\mathrm{C}$ an $a_{1}$, Profit: 20

2. $a_{1}$ verkauft $\mathrm{A}$ an $a_{2}, a_{2}$ verkauft $\mathrm{B}$ an $a_{3}, a_{3}$ verkauft $\mathrm{C}$ an $a_{4}$, Profit: 10

Derselbe Sachverhalt wird in ähnlicher Form in [Schier \& Fischer 96] dargestellt. Eine ausführliche, mathematische Darstellung findet sich in [Malich 94]. 


\section{Simulated Trading zwischen Speditionen}

Der oben skizzierte Ansatz läßt sich nicht analog auf die Kooperation zwischen verschiedenen Unternehmen übertragen. Die Speditionen sind in erster Linie an ihrem eigenen Profit interessiert und der Gesamtgewinn des Verbundes ist aus der lokalen Sicht der Speditionen zweitrangig, insbesondere, wenn der Gesamtgewinn nicht auf die Teilnehmer umgelegt wird. Eine Spedition wird somit bevorzugt einem Handel zustimmen, wenn sie davon profitiert. Teilnehmer, die ausschließlich nach rationalen, spieltheoretischen Kriterien entscheiden, werden nur einen profitablen Handel abschließen. Deshalb muß das Modell dahingehend erweitert werden, daß es Ausgleichszahlungen für Auftragsweitergabe vorsieht. Durch monetären Nutzenausgleich können lokale Verschlechterungen kompensiert werden, die erforderlich sind, um die Gesamtlösung zu optimieren. Ein weiteres Problem besteht darin, daß Verschlechterungen in der globalen Lösung nur dann für jede einzelne Spedition profitabel sind, wenn Ausgleichszahlungen von "außerhalb" geleistet werden, z.B. wenn der Koordinator über eigene finanzielle Ressourcen verfügt, mit denen Tauschgeschäfte, die die Gesamtlösung verschlechtern, subventioniert werden können.

In unserem Ansatz statten wir daher den LDZ-Agenten mit eigenen finanziellen Ressourcen aus, die es ihm ermöglichen, auf den Trading Prozess Einfluß zu nehmen. Der LDZ-Agent fungiert bei jedem Handel im Trading Prozess als Vermittler, der eine positive oder negative Provision berechnet. Wenn ein Handel für beide Partner vorteilhaft ist, wird ein Teil des Profits vom LDZ-Agenten einbehalten. Aus diesen Mitteln können im späteren Verlauf unvorteilhafte Geschäfte subventioniert werden, wenn sie die Gesamtlösung verbessern oder, wenn versucht wird, durch eine temporäre Verschlechterung der Gesamtlösung neues Optimierungspotential zu erschließen.

In diesem Szenario sind die teilnehmenden Speditionen autonome und eigennützige Agenten. Demnach spiegeln die Gebote nicht notwendigerweise ihre tatsächliche Nutzenbewertung wider, sondern können bereits einen, nach strategischen Erwägungen bestimmten, Profit enthalten. Die Gebote müssen somit unter spieltheoretischen Gesichtspunkten betrachtet werden.

Im folgenden untersuchen wir drei Szenarien, in denen sich die Strategien der beteiligten Speditionen durch unterschiedliche Kooperationsbereitschaft auszeichnen. In Abhängigkeit von diesen Strategien loten wir die Spielräume des Managers aus.

Im weiteren seien $k(a, o)$ die tatsächlichen Kosten, die dem Agenten $a$ für Auftrag $o$ anfallen, von denen die Gebote abweichen können. Mit $p(a, b)$ bezeichnen wir die Zahlung die Agent $a$ erhält bzw. leisten muß, falls das Gebot $b$ zugeordnet wird. Wenn ein Auftrag abgegeben wird, zahlt der Agent maximal die angegebenen Kosten, die die tatsächlichen Kosten noch unterschreiten können. Es gilt also $p(a, b) \leq c(b) \leq k(a, o)$. Umgekehrt gilt im Falle einer Auftragsübernahme $p(a, b) \geq c(b) \geq k(a, o)$. Der (möglicherweise negative) Profit des Brokers für die Zuordnung einer Kante $e\left(b_{i k}, b_{j l}\right)$ beträgt also $p^{B}\left(b_{i k}, b_{j l}\right):=p\left(a_{i}, b_{i k}\right)-p\left(a_{j}, b_{j l}\right)$.

Die Agenten sind "ehrlich" und uneigennützig Die Bieter geben ihre exakten Kosten für Kauf und Verkauf von Aufträgen an. Falls profitable Händel 
abgeschlossen werden schöpft der Stock Manager die gesamte Differenz ab und erhält so einen großen Spielraum, um weniger profitable Verkäufe zu subventionieren. Insbesondere kann ein Handel, der die Gesamtlösung verschlechtert, ohne Verluste für den Manager rückgängig gemacht werden.

In diesem Fall gilt also $p(a, b)=c(b)=k(a, o)$ und $p^{B}\left(b_{i k}, b_{j l}\right)=k\left(a_{i}, o\right)-k\left(a_{j}, o\right)$ mit $o=o\left(b_{i k}\right)=o\left(b_{j l}\right)$.

Die Agenten sind "ehrlich" und eigennützig Die Bieter geben ihre exakten Kosten für Kauf und Verkauf von Aufträgen an. Der Stock Manager behält jedoch nur einen Teil der Differenz der Gebote als Vermittlungsprovision und gibt den Rest als Gewinn an die Bieter weiter. In diesem Fall ist der Spielraum des Managers geringer und schlechte Händel können nur mit Verlust rückgängig gemacht werden.

Hier gilt $p(a, b)$ ist verschieden von den Kosten $c(b)=k(a, o)$. Die Differenz zwischen $p(a, b)$ und $c(b)$ hängt von der Strategie des Brokers ab. Wir haben drei prinzipielle Optionen.

- Die Gewinne der Agenten werden durch einen konstanten Zuschlag realisiert, d.h. $p\left(a, b_{\text {buy }}\right)=c\left(b_{\text {buy }}\right)+k$ bzw. $p\left(a, b_{\text {sell }}\right)=c\left(b_{\text {sell }}\right)-k$ für ein $k>0$.

- Die Gewinne werden durch eine prozentuale Anhebung der Gebote realisiert, d.h. $p\left(a, b_{\text {buy }}\right)=k \cdot c\left(b_{\text {buy }}\right)$ bzw. $p\left(a, b_{\text {sell }}\right)=\frac{1}{k} \cdot c\left(b_{\text {sell }}\right)$ für ein $k>1$.

- Die Differenz zwischen Verkauf- und Kaufgebot (falls positiv) wird zwischen Käufer, Verkäufer und Manager verteilt, d.h. $p\left(a, b_{b u y}\right)=c\left(b_{b u y}\right)+\frac{\Delta}{3}$ und $p\left(a, b_{\text {sell }}\right)=c\left(b_{\text {sell }}\right)-\frac{\Delta}{3}$ für $\Delta=c\left(b_{\text {sell }}\right)-c\left(b_{\text {buy }}\right)>0$. Falls die Differenz negativ ist, wird auf einen der obigen Fälle zurückgegriffen.

Die Agenten sind "unehrlich" und eigennützig Sie geben Gebote ab, die bereits ihren Gewinn enthalten. Also $c\left(b_{\text {sell }}\right)<k\left(a, o\left(b_{\text {sell }}\right)\right)$ bzw. $c\left(b_{\text {buy }}\right)>$ $k\left(a, o\left(b_{b u y}\right)\right)$. Der Broker kann weniger Gewinn abschöpfen bzw. muß mehr draufzahlen. Der Spielraum des Brokers sinkt. Die Bieter können ihre Gebote um einen konstanten Summanden oder Faktor erhöhen oder erniedrigen. Der Broker kann entweder die Gesamtdifferenz der Gebote, die ja bereits Gewinne enthalten, einbehalten, oder wie im vorherigen Fall, lediglich Teilbeträge einbehalten.

\section{Parameter für die kompetitive Optimierung}

Um das vorhandene Optimierungspotential voll auszuschöpfen sind unterschiedliche Parameter aufeinander abzustimmen. Folgende Parameter für den Trading Prozess sind zu betrachten:

Finanzielle Ressourcen des Brokers: Die Summe, die der Broker am Beginn des Tradings zur Verfügung hat, ist begrenzt, da sie von den Teilnehmern bereitgestellt werden muß. Allerdings muß sie ausreichend hoch gewählt werden, um dem Broker den nötigen Spielraum für die Optimierung zu ermöglichen. 
Subventionsstrategie: Die Strategie des Brokers bestimmt den Anteil, den der Broker als Provision einbehält, bzw. mit dem unvorteilhafte Tauschgeschäfte subventioniert werden. Der Anteil muß hoch genug sein um die Flexibilität des Brokers zu erhalten, sollte jedoch den Teilnehmern angemessene Profite ermöglichen.

Kapitalentwicklung des Brokers: Wenn der Broker ein LDZ repräsentiert, das mehr als eine virtuelle Organisationseinheit im Speditionsverbund darstellt, kann es sinnvoll sein, den Broker im Laufe der Optimierung Überschüsse erwirtschaften zu lassen. Im Idealfall kann das LDZ ausschließlich durch die Optimierungsgewinne finanziert werden. Gegen ein hohes Gewinnstreben des Brokers spricht jedoch der dadurch eingeschränkte Optimierungsspielraum.

Strategien der Bieter: Da die Bieter rational handeln, streben sie möglichst hohe Profite an. Um jedoch überhaupt eine Optimierung zu ermöglichen, ist es erforderlich, daß die Speditionsagenten mit moderaten Gewinnvorstellungen antreten.

\section{Zusammenfassung und Ausblick}

Wir haben einen multiagentenbasierten Ansatz zur Unterstützung der Geschäftsprozesse in einem kooperativen Verbund von Transportunternehmen vorgestellt, der es ermöglicht, die Effizienz der Transportdienstleistungen der beteiligten kleinen und mittleren Unternehmen zu steigern und damit ihre Wettbewerbsfähigkeit zu erhöhen. Zu diesem Zweck wurde das verteilte Simulated Trading Optimierungsverfahren konzeptuell so erweitert, daß es in kompetitiven Domänen eingesetzt werden kann und Optimierung zwischen rationalen Agenten ermöglicht, die Entscheidungen nach spieltheoretischen Kriterien treffen.

Wir planen, die TeleTruck-CC Implementierung anhand von Testdaten aus der Praxis zu evaluieren. Zielsetzung ist dabei, die Parameter aus dem vorigen Abschnitt derart zu wählen, daß eine möglichst effektive Optimierung vorgenommen werden kann.

Im Rahmen eines Partner-Projektes Platform werden Aspekte der intermodalen Transportplanung untersucht [Funk et al. 98]. Künftige Arbeiten werden sich mit der Integration von kooperativer, speditionsübergreifender Transportplanung und intermodaler Transportplanung befassen.

\section{Danksagung}

Wir danken den Mitarbeitern der Spedition Konz und des Logistischen Dienstleistungs Zentrum des Saarlandes für die Unterstützung bei der Modellierung der Unternehmensabläufe im Speditionsgewerbe. Das TeleTruCK-CC Projekt wird von der Saarländischen Landesregierung gefördert. 


\section{Literatur}

[Bürckert et al. 98] H.-J. Bürckert, K. Fischer, and G. Vierke. Transportation Scheduling with Holonic MAS - The TeleTruck Approach. In: Proceedings of the Third International Conference on Practical Applications of Intelligent Agents and Multiagents (PAAM'98), 1998.

[Carroué 97] Laurent Carroué. La ruinease maladie $d u \ll$ tout-routier $\gg-$ Une Europe des transports menacée d'embolie. Le Monde Diplomatique, pp. 18-19, Dezember 1997.

[Fischer 98] K. Fischer. TeleTruck: Ein Online-Dispositionssystem für Speditionen. Informationstechnik und Technische Informatik, 4(40):30-33, 1998.

[Funk et al. 98] P. Funk, G. Vierke, and H.-J. Bürckert. A Multi-Agent Systems Perspective on Intermodal Transport Chains. Technical Memo TM98-06, DFKI, 1998.

[Gerber et al. 99] C. Gerber, J. Siekmann, and G. Vierke. Flexible Autonomy in Holonic Agent Systems. In: Proceedings of the 1999 AAAI Spring Symposium, 1999. To appear.

[Klempp 98] H. Klempp. Ökologie im Straßengütertransport. Verlagsbeilage Logistik und Transportmanagement, Frankfurter Allgemeine Zeitung Nr. 203, 2. September, p. B10, 1998.

[Malich 94] M. Malich. Simulated Trading - Ein paralleles Verfahren zur Lösung von kombinatorischen Optimierungsproblemen. PhD thesis, Universität zu Köln, 1994.

[Schier \& Fischer 96] D. Schier and K. Fischer. Ein Multiagentenansatz zum Lösen von Fleet-Scheduling-Problemen. DFKI Dokument D-96-01, DFKI GmbH, Saarbrücken, 1996. 

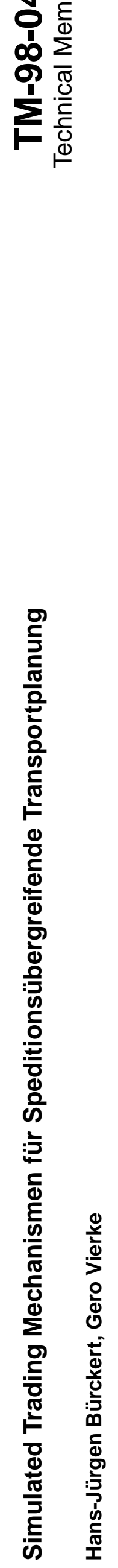